\title{
Retos 4.0: un manifiesto a considerar para la 4RI
}

\author{
doi: 10.5281/zenodo.4654853
}

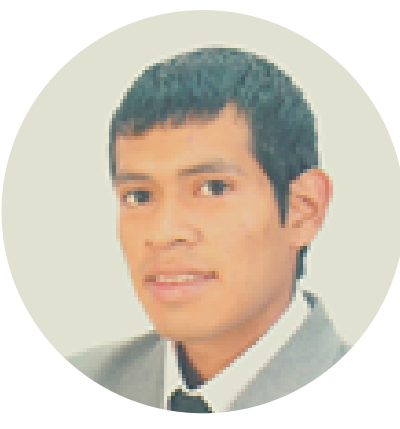

\section{WILDO SIANCAS}

Psicólogo por la Universidad San Ignacio de Loyola. Cuenta con estudios de maestría en Educación con mención en Psicología educativa. Se ha desempeñado como docente en organizaciones no gubernamentales orientadas al sector educativo y en psicología clínica infantil. Consultor independiente en metodología de la investigación. Miembro de la Sociedad Secular Humanista del Perú.

wildo_esmith_14@hotmail.com

La Cuarta Revolución Industrial permite que el avance exponencial de la ciencia y tecnología tenga un impacto cada vez mayor en las dimensiones orgánicas, psíquicas, culturales e interactivas de la naturaleza humana; sin embargo, los retos que trae consigo son cada vez más complejos y transversales. Por ende, resulta idóneo analizarlos en los ámbitos biológicos, psicológicos, sociales y contextuales del ser humano a fin de generar espacios de diálogo en las futuras generaciones.

\section{La biología a merced de la ciencia y tecnología}

Las tecnologías NBIC (Nanotecnología, Biotecnología, Tecnologías de la información y Ciencias cognitivas) han impulsado el desarrollo de la Industria 4.0; sin embargo, cuando se hallan a merced de conflictos e intereses personales, sus aplicaciones adquieren un potencial iatrogénico en la condición humana (Bunge, 2019). En las neurociencias, el uso excesivo del término neuro, ha permitido el auge de pseudodisciplinas carentes de solidez científica (Cumpa-Valencia, 2019; Corredor \& Cárdenas, 2017; García-Albea, 2011). Por ende, se necesita analizar la interdisciplinariedad, la continuidad de sus hallazgos y la autonomía epistemológica de sus disciplinas (Restrepo, 2020). Caso contrario, del uso acrítico de sus conclusiones, fluirán argumentos pseudocientíficos traducidos en neuromitos (Calderón, 2017), afirmaciones que afectan tanto al docente, como al alumno y la metodología educativa (Painemil et al., 2021).

Por otro lado, la búsqueda de una singularidad tecnológica fuera del control humano involucraría ciertos desafíos en el desarrollo de la inteligencia artificial, por ejemplo, alteraciones en algoritmos de ejércitos robóticos, accidentes en infraestructuras digitales, armas programables de destrucción masiva o aumento del desempleo
(Turchin \& Denkenberger, 2018).

En cuanto a la nanotecnología, sus retos yacen en materia de sostenibilidad ecológica y sanitaria (Foladori, 2012). Ante ello, la bioacumulación de residuos en la cadena trófica y la nanotoxicología se erigen como potenciales riesgos (Delgado, 2007; Reyes et al., 2009). En ese sentido, la bioingeniería también necesita una reflexión ética en la regulación de sus hallazgos. El desarrollo de biotecnologías con fines civiles y militares requiere una precisión ética en sus aplicaciones bélicas, ya que grandes avances ameritan mayores responsabilidades (Ramirez, 2016; Michavila \& Granda, 1994), como es el caso de la ingeniería genética. En este contexto aparece el biohacking, que surge como una necesidad sociocultural de una ciencia más abierta y democratizada (Wexler, 2017); sin embargo, cuando la edición genética traspasa las fronteras éticas, lejos de suponer un beneficio, representa un riesgo si no es monitoreada de manera regular (Cique, 2017; Wolinsky, 2016), por ello, la eugenesia se presenta como un paradigma necesario de precisar y abordar desde la bioética (Sagols, 2010).

\section{Los puntos opacos de la psicología}

En cuanto a las ciencias del comportamiento, el desarrollo de la 4RI implica ciertos desafíos que ameritan precisar. A nivel cognitivo los individuos presentan sesgos y atajos heurísticos en el procesamiento de la información de su entorno (Kahneman, 2012), por ende, las posibilidades de omitir, distorsionar o manipular la información aumentan (Benítez, 2019; Gómez, 2018; Mendoza, 2020).Asimismo, la capacidad predictiva de los algoritmos cognitivos en las decisiones de masas homogéneas dedicadas al consumo excesivo genera la capitalización de la atención mediante resultados de búsqueda que apartan al usuario de una 
información clara, concisa y objetiva (Celis, 2017; Figueroa, 2018).

Como resultado, se manifiesta una semántica afectiva según el desarrollo volitivo del usuario. Por lo tanto, las emociones y la ofensa permiten la manifestación de la violencia en redes digitales (Kaiser, 2020; Martín, Pazos, Montilla \& Romero, 2016). Siendo así el Ciberbullying, los discursos de odio e incluso la manipulación de la identidad digital, aspectos que urgen ser abordados $y$ regulados (González-Ramírez \& López-Gracia, 2018; Betts, 2016; Miró, 2016; Díaz, 2015; Prieto et al., 2015).

Por otro lado, a nivel conductual, las adicciones a las nuevas tecnologías tendrán repercusiones en el comportamiento del usuario, tales como: Síndromes de abstinencia prolongados, tolerancia al uso abusivo de los dispositivos, bajo nivel de respuesta al tratamiento e incluso modificaciones en circuitos neuronales (Rodríguez et al., 2012). Por tanto, el abuso de los videojuegos, la dependencia al Internet, el miedo a estar sin celular o incluso, el desarrollo de nudges informáticos, se han vuelto el costo a pagar en el desarrollo de algoritmos informáticos capaces de modificar conductas mediante reforzadores digitales como likes, views, matches, followers (Peirano, 2019; Thaler \& Sunstein, 2017).

\section{La búsqueda de una reorganización social mediática}

A nivel social, la industria 4.0 también trae consigo una serie de retos en el imaginario colectivo de sus habitantes. Dentro del campo del desarrollo económico, la desigualdad en el acceso a los recursos, el clientelismo político y el asistencialismo han generado una brecha socioeconómica en sus miembros (Adelantado \& Scherer, 2008), aspectos que agravan el acceso a las tecnologías exponenciales y conllevan a la gestación de sistemas dualistas de estratificación social (Alva de la Selva, 2015; Tezanos, 2002). Como resultado, el ciberactivismo se manifiesta como un espacio digital y social, en donde la ciudadanía busca participar en los cambios estructurales de sus gobiernos (Cortes \& Garzón, 2017; Soengas-Pérez \& Assif, 2017; Manrique, 2016). Esto trae consigo el auge de corrientes como el postmodernismo y el relativismo científico, cuyos discursos permiten la gestación del pensamiento pseudocientífico e ideas conspiranoicas en contextos digitales (Jaimes, 2020; López de Pomar, 2020; Gallo, 2019; Cendrós \& Urdaneta, 2006). Estos entornos, a nivel histórico- cultural, gestan pseudoideologías capaces de acrecentar tanto la polarización en redes sociales como la concentración de medios e incluso, la desinformación mediante fake news y bulos (Badillo, 2019; Rodríguez-Fernández, 2019; Lozada, 2004; Chomsky \& Ramonet, 1993). Aspectos que afectan el desarrollo de políticas públicas, abusan del poder, hacen uso de la violencia y atentan contra la democracia (Hadit \& Lukianoff, 2019; Chomsky, 2017). Un contrato social afectado por la proliferación de discursos mediáticos a través de influencers, cuya opinión simple, sesgada y parcializada se impone a pesar de los hechos y evidencias científicas (Garibay et al., 2019; Dumortier, 2009; Yearly, 1993).

\section{La construcción de brechas contextuales}

Respecto al impacto de la Industria 4.0 en las interacciones de cada una de sus instituciones sociales, como la familia, la escuela, el trabajo e incluso el ambiente, aumentan las demandas y exigencias hacia éstas. Por ejemplo, la familia presenta serios retos en la digitalización de las interacciones entre sus integrantes, hecho que se evidencia en el aislamiento comunicativo entre sus miembros y la falta de supervisión en el uso de los entornos virtuales (Barrera \& Duque, 2014; Hernández et al., 2014). Para este caso la supervisión parental requiere de mediación y estilos de control según la forma en cómo se comunican sus miembros (López-Sánchez \& García del Castillo, 2017). Razón por la cual, los estilos de crianza y los tipos de educación parental requieren aunar esfuerzos con el sistema educativo al momento de optimizar la formación ciudadana y cívica de futuros agentes de cambio.

Por otro lado, dentro del marco educativo, la mercantilización de este sistema ocasiona una hegemonía cultural que erige masas acríticas, pasivas y dependientes (Chomsky, 2014). Así pues, el acceso a la información y gestión del conocimiento se convierten en factores clave, tanto del pensamiento crítico como de la producción científica, en las entidades académicas de nivel básico y superior. Sin embargo, existen brechas digitales que dificultan el aprendizaje significativo tales como: infraestructuras virtuales deficientes, equipos costosos, escuelas remotas con bajo acceso a Internet e incluso, monopolios editoriales cuyas revistas patologizan el factor de impacto (Luchilo, 2019; Hernández, 2017; Santos \& Fernandez-Ríos, 2016; Münch, 2015; Cabrero, 2007). El surgimiento de revistas depredadoras, la expansión de movi- 
mientos de hardware/software libres o el uso metabuscadores informales, se vuelven el medio de reclamo por parte de los intelectuales y la academia. Ello con el fin de conseguir una ciencia más abierta que considere la participación ciudadana, tanto del sector público como privado, en la cultura científica. (García-Puente, 2019; Himmelstein et al., 2018; Bertoglia \& Águila, 2018; García \& Rendueles, 2014; Culebro et al., 2006; López, 2005).

En cuanto al ámbito laboral, el reto de las instituciones es la adaptación organizacional hacia la transformación digital. Así pues, el extractivismo rentista y el manejo deficiente de los recursos ocasionan la corrupción estructural de las corporaciones (Gudynas, 2017; Sandoval, 2016). Como consecuencia, se perpetuán relaciones nocivas de poder entre la organización y el trabajador (Lastra, 2017; Jované, 2015). Aspecto que necesita un marco legal regulatorio que considere los riesgos psicosociales asociados al teletrabajo en caso de ambigüedad de roles, baja autonomía, sobre-extensión horaria y baja conciliación trabajovida (Rubbini, 2012; Alonso \& Cifre, 2002). De misma manera, la informalidad del mercado negro de la Deep web o la especulación de burbujas inflacionarias de criptomonedas serán herramientas económicas capaces de superar los altibajos de la regulación estatal (Bedecarratz, 2018; Navas, 2015; Aquino, 2008).

A nivel medioambiental, el reto de la sostenibilidad tecnológica es una de las tareas que aún que-

\section{Referencias}

Adelantado J., \& Scherer, E. (2008). Desigualdad, democracia y políticas sociales focalizadas en América latina. Estado, Gobierno y Gestión Pública, 11(5), 117-134. https://revistaeggp.uchile.cl/index.php/REGP/article/view/141 $\underline{43}$.

Alonso M., \& Cifre, E. (2002). Teletrabajo y Salud: un nuevo reto para la psicología. Papeles del Psicólogo, 83(8), 55-61. https: //www. redalyc. org/articulo. oa? id $=77808308$.

Alva de la Selva, A. (2015). Los nuevos rostros de la desigualdad en el siglo XXI:la brecha digital. Revista Mexicana de Ciencias Políticas y Sociales, 60(223), 265-286. http://www. revistas.unam.mx/index.php/rmcpys/article/view/4 5387.

Aquino, R. (2008). El mercado negro de internet. Revista Digital Universitaria, 9(4), 3-9. http://www.revista.unam.mx/vol.9/num4/art24/int24.htm.

Badillo, A. (2019). La sociedad de la desinformación: propaganda, «fake news» y la nueva geopolítica de la información. Fundación Real Instituto Elcano. http://www.realinstitutoelcano.org/wps/portal/rielcano es/cont enido?

WCM GLOBAL CONTEXT=/elcano/elcano es/zonas es/len gua +y+cultura/dt8-2019-badillo-sociedad-de-desinformacionpropaganda-fake-news-y-nueva-geopolitica-de-informacion. dan pendientes. Fenómenos como la obsolescencia programada, el manejo de desechos sólidos, la pleotropía en los cultivos transgénicos o incluso las implicancias del cambio climático en la salud mental de la población, son aspectos a tomar en cuenta frente al remanente ecológico que nos espera (Ordoñez-Iriarte, 2020; Clayton, 2019; Martínez \& Porcelli, 2016; Bustos, 2009; Herbert et al., 2006). Una tarea colectiva aún pendiente en las entidades públicas y privadas que tienen como objetivo el desarrollo sostenible de sus organizaciones.

\section{Acotaciones finales}

En síntesis, la Cuarta Revolución Industrial involucra una serie de retos y nuevas formas de difusión en el manejo de la información. Ante ello, las ciencias naturales, a merced de las tecnologías exponenciales, necesitan replantear los límites de la mejora humana. Por consiguiente, la ciberpsicología necesita precisar cómo la información se genera, procesa y construye al momento en que los ciudadanos elaboran un discurso propio de modo que ofrezca una narrativa histórico-cultural que cuestione el postmodermisno y las ideologías pseudocientificas de la civilización del espectáculo afin de tender puentes en las brechas contextuales propias de la realidad humana y sus instituciones sociales. Caso contrario, el arquetipo high tech-low life, propio del género cyberpunk, podría ser la narrativa imperante en nuestro paradigma colectivo.

Barrera D., \& Duque, L. (2014). Familia e internet: consideraciones sobre una relación dinámica. Revista Virtual Universidad Católica del Norte, 41(4), 30-44.

http://www. redalyc.org/articulo.oa?id=194229980004.

Bedecarratz, F. (2018). Riesgos delictivos de las monedas virtuales: Nuevos desafíos para el derecho penal. Revista chilena de derecho y tecnología, 7(1), 79-105. https://scielo.conicyt.cl/scielo.php? script $=$ sci abstract\&pid=S071925842018000100079\&lng=es\&nrm=iso.

Benítez, L. (2019). Ética y transparencia para la detección de sesgos algorítmicos de género. Estudios Sobre El Mensaje Periodístico, 25(3), 1307-1320. https://doi.org/10.5209/esmp.66989.

Bertoglia, M. \& Águila, A. (2018). Revistas depredadoras: una nueva amenaza a las publicaciones científicas. Revista médica de Chile, 146(2), 206-212. https://scielo.conicyt.cl/scielo.php? script=sci arttext\&pid=S0034-98872018000200206.

Betts, L. (2016). Cyberbullying. Palgrave Macmillan.

Bunge, M. (2019). Promesas y amenazas del adelanto tecnológico. Instituto de Extrapolítica y Transhumanismo. https://extrapolitica.ssh.org.pe/wpcontent/uploads/2019/10/Bunge-Mario-Promesas-yAmenazas-del-Adelanto-Tecnol\%C3\%B3gico-IET.pdf. 
Bustos, C. (2009). La problemática de los desechos sólidos. Economía, 34(27), 121-144. https://www.redalyc.org/pdf/1956/195614958006.pdf.

Cabrero, J. (2007). Las necesidades de las TIC en el ámbito educativo: oportunidades, riesgos y necesidades. Tecnología y Comunicación Educativas, 21(45), 4-19. https://biblat.unam.mx/es/revista/tecnologia-y-comunicacioneducativas/articulo/las-necesidades-de-las-tic-en-el-ambitoeducativo-oportunidades-riesgos-y-necesidades.

Calderón, L. (2017). La Neurociencia: una postura crítica frente al "boom" por la "neuro". CES Psicología, 10(1), 1-3. http://www. scielo.org.co/scielo.php? script=sci arttext\&pid=S2011-30802017000100001.

Celis, C. (2017). Economía de la atención y visión maquínica: hacia una semiótica asignificante de la imagen. Hipertextos, 5(7), 41-55.

https://revistas.unlp.edu.ar/hipertextos/article/view/7759.

Cendrós, J., \& Urdaneta, E. (2006). Internet icono del postmodernismo. Frónesis, 13(2) http://ve.scielo.org/scielo.php?script=sci arttext\&pid=S1315$\underline{62682006000200006 .}$.

Chomsky, N. (2017). Estados fallidos. Ediciones B.

Chomsky, N. (2014). La (des)educación. Crítica.

Chomsky N., \& Ramonet, I. (1993). Cómo nos venden la moto. Icaria. Cique, A. (2017). «Biohacking» y «biohackers»: amenazas y oportunidades. Paseo de la Castellana: Instituo Español de Estudios

Estratégicos. http://www.ieee.es/Galerias/fichero/docs opinion/2017/DIEEE 093-2017 Biohcking_CiqueMoya.pdf.

Clayton, S. (2019). Psicología y Cambio Climático. Papeles del Psicólogo, 40(3),

167-173. https://doi.org/10.23923/pap.psicol2019.2902.

Corredor, K., \& Cárdenas, F. (2017). Neuro-«lo que sea» : inicio y auge de una pseudociencia para el siglo XXI. Revista Latinoamericana de Psicología, 49(2), 89-90. https://www.redalyc.org/articulo. oa? id=80551191001.

Cortes D., \& Garzón, T. (2017). El ciberactivismo en las revoluciones posmodernas. Revista de Estudios en Seguridad Internacional, 3(1), 103-125. https://seguridadinternacional.es/resi/html/el-ciberactivismoen-las-revoluciones-posmodernas/.

Culebro, M., Gómez, W., \& Torres, S. (2006). Software libre vs. Software propietario. Creative commons.

Cumpa-Valencia, M. (2019). Usos y abusos del término "neurociencias": una revisión sistemática en revistas indexadas Scielo. Revista ConCiencia EPG, 4(1), 30-67. https://revistaconcienciaepg.edu.pe/ojs/index.php/55551/articl e/view/12.

Delgado, G. (2007). Incertidumbres de la nanotecnología: riesgos ambientales y salud. Ingeniería de Recursos Naturales y del Ambiente, 6(1), 47-61. https://www.redalyc.org/articulo.oa? $i \mathrm{~d}=231120826005$.

Díaz, J. (2015). una aproximación al concepto de discurso del odio. Revista Derecho del Estado, 34(Julio), 77-101. https://papers.ssin.com/sol3/papers.cfm? abstract id=2637492.

Dumortier, F. (2009). Facebook y los riesgos de la «descontextualización» de la información. IDP. Revista de Internet, Derecho y Política, 9(Diciembre), 25-41. https://www.redalyc.org/articulo.oa?id=78813254009.

Echeburúa, E. (2012). Factores de riesgo y factores de protección en la adicción a las nuevas tecnologías y redes sociales en jóvenes y adolescentes. Revista Española de Drogodependencias, 37(4), 435-447. https://www.aesed.com/descargas/revistas/v37n4 5.pdf.
Figueroa, M. (2018). Los algoritmos de Internet y el monopolio sociocultural de los buscadores en la entrega de datos: opciones para salir de la burbuja. Estelí: Ábaco en red. Una ruta alternativa popular en educación.

https://abacoenred.com/wpcontent/uploads/2018/12/contenido-

2 algoritmosdebusqueda y-burbujasdefiltros. pdf.

Foladori, G. (2012). Riesgos a la salud y al medio ambiente en las políticas de nanotecnología en América Latina. Sociológica, 27(77), 143-180. http://www.scielo.org.mx/scielo.php? script=sci arttext\&pid=S0187-01732012000300005.

Gallo, A. (2019). Teorías de la conspiración: de la paranoia al genocidio. Estudios Humanísticos. Filología, 41(11), 217-243. https://dialnet.unirioja.es/servlet/articulo?codigo $=7200694$.

García D., \& Rendueles, C. (2014). Abierto, libre... y público. Los desafíos políticos de la ciencia abierta. Argumentos de razón técnica: Revista española de ciencia, tecnología y sociedad, y filosofía de la tecnología, 17(3), 45-64. https://dialnet.unirioja.es/servlet/articulo?codigo=4935407.

García-Albea, J. (2011). Usos y abusos de lo "Neuro". Revista de Neurología, $52(10)$ https://www.neurologia.com/articulo/2010313.

García-Puente, M. (2019). La epidemia de las revistas depredadoras. Revista pediatría Atención Primaria, 21(81), 81-85. http://scielo.isciii.es/scielo.php? script=sci arttext\&pid=S113976322019000100019 .

Garibay, I., Matzaris, A., Rajabi, A., \& Taylor, C. (2019). Polarización en redes sociales ayuda a que los influencers tengan más influencia: Análisis y dos estrategias de inoculación. Perfiles de ingeniería, 15(15), 53-67. https://revistas.urp.edu.pe/index.php/Perfiles Ingenieria/articl e/view/3379.

Gómez, J. (2018). Segmentación, sesgo y normas sociales en la programación. Avatares de la comunicación y la cultura, 15(Junio),

1-14. https://publicaciones.sociales.uba.ar/index.php/avatares/articl e/view/4889.

González-Ramírez, T., \& López-Gracia, A. (2018). La identidad digital de los adolescentes: usos y riesgos de las Tecnologías de la Información y la Comunicación. Revista Latinoamericana de Tecnología Educativa, 17(2), 73-85. https://relatec.unex.es/article/view/3319.

Gudynas, E. (2017). Extractivismos y corrupción en América del Sur. Estructuras, dinámicas y tendencias en una íntima relación. Revista de Ciencias Sociales y Humanas, 10(10), 73-87. https://www.redalyc.org/jatsRepo/5535/553559586007/html/in dex.html.

Hadit J. \& Lukianoff, G. (2019). La transformación de la mente moderna. Deusto.

Herbert, M., García, J., \& García-G, M. (2006). Alimentos transgénicos: Incertidumbres y riesgos basados en evidencias. Acta Académica, 39(Noviembre), 129-145. http://revista.uaca.ac.cr/index.php/actas/article/view/424.

Hernández, A., López, P., \& Sánchez, S. (2014). La comunicación en la familia a través de las TIC. Percepción de los adolescentes. Pulso: Revista de educación, 37(2), 35-58. https://dialnet.unirioja.es/servlet/articulo?codigo=4954346.

Hernández, R. (2017). Impacto de las TIC en la educación: Retos y Perspectivas. Propósitos y Representaciones, 5(1), 325-347. http://dx.doi.org/10.20511/pyr2017.v5n1.149.

Himmelstein, D., Rodríguez, A., Levernier, J., Munro, T., Reid, S., Greshake, B., \& Greene, C. (2018). Sci-Hub provides access to nearly all scholarly literature. eLife, 7(32822), 1-22. https://elifesciences.org/articles/32822.

Jaimes, F. (2020). Pseudociencias: Una manifestación del 
pensamiento errado. Instituto de Extrapolítica y Transhumanismo. https://extrapolitica.ssh.org.pe/wpcontent/uploads/2020/09/Jaimes-Freddy-2020-

Pseudociencia-Una-manifestaci\%C3\%B3n-del-pensamientoerrado-IET.pdf.

Jované, J. (2015). El poder de dirección del empleador vs. el acceso de los medios tecnológicos e informáticos dentro de la empresa. Revista VIA IURIS, 18(3), 47-71. https://www. redalyc.org/articulo.oa?id=273944646003.

Kahneman, D. (2012). Pensar rápido, pensar despacio. Debate. Kaiser, A. (2020). La Neoinquisición. Planeta.

Lastra, J. (2017). Rodríguez Escanciano, Susana, Poder de control empresarial, sistemas tecnológicos y derechos fundamentales de los trabajadores. Boletín Mexicano de Derecho Comparado, 50(149), 1069-1073.

http://www.scielo.org. mx/scielo.php? script=sci arttext\&pid=S0041-86332017000201069.

López, J. (2005). Participación ciudadana y cultura científica. Arbor, 181(715),

351-362. http://arbor.revistas.csic.es/index.php/arbor/article/view/417.

López de Pomar, F. (2020). El virus conspirativo: Una vacuna. Instituto de Extrapolítica y Transhumanismo. https://extrapolitica.ssh.org. pe/wp-

content/uploads/2020/05/Lopez-de-Pomar-Fabrizio-El-virusconspirativo-una-vacuna-IET.pdf.

López-Sánchez, C., \& García del Castillo, J. (2017). La familia como mediadora ante la brecha digital: repercusión en la autoridad. Revista Colombiana de Ciencias Sociales, 8(1), pp. 108-124. http://dx.doi.org/10.21501/22161201.1928.

Lozada, M. (2004). El otro es el enemigo: imaginarios sociales y polarización. Revista Venezolana de Economía y Ciencias Sociales, 10(2), 195-209. https://www.redalyc.org/articulo.oa? $i \mathrm{~d}=17710214$.

Luchilo, L. (2019). Revistas científicas: oligopolio y acceso abierto. Revista Iberoamericana de Ciencia, Tecnología y Sociedad, 14(40), 41-79. https://www.redalyc.org/jatsRepo/924/92459230002/html/inde x.html.

Manrique, L. (2 al 4 de noviembre de 2016). Ciberactivismo en Facebook: una estrategia didáctica para acciones colectivas locales [Sesión de conferencia]. Humanidades digitales, diálogo de saberes y practicas colaborativas en red:Cátedra UNESCO de comunicación, Bogotá, Colombia. https://www.javeriana.edu.co/unesco/humanidadesDigitales/p. onencias/IV 115.html.

Martín, A., Pazos, M., Montilla, M., \& Romero, C. (2016). Una modalidad actual de violencia de género en parejas de jóvenes: Las redes sociales. Educación XX1, 19(2), 405-429. http://www.redalyc. org/articulo.oa?id=70645811017.

Martínez, A., \& Porcelli, A. (2016). Consumo (In) sostenible: nuevos desafíos frente a la obsolescencia programada como compromiso con el ambiente y la sustentabilidad. Ambiente $y$ Sostenibilidad, 49(6), 105-135. https://bibliotecadigital.univalle.edu.co/handle/10893/11521.

Mendoza, D. (2020). Racismo y roles de género, conductas perpetuadas en algoritmos de inteligencia artificial. Coloquio, 65(11),

131-135.

http://revistas.uazuay.edu.ec/index.php/coloquio/article/view/3 $\underline{33}$.

Michavila, B., \& Granda, J. (1994). Consideraciones para la aplicación de tecnologías duales. Cuadernos de estrategia, 67(4), 47-72. https://dialnet.unirioja.es/servlet/articulo? codigo $=2778087$.

Miró, F. (2016). Taxonomía de la comunicación violenta y el discurso del odio en Internet. IDP. Revista de Internet, Derecho y
Política, 22(Junio),

82-107.

https://www. redalyc. org/articulo.oa?id=78846481007.

Münch, R. (2015). El mecanismo de monopolio en la ciencia.

Literatura: teoría, historia, crítica, 17(2), 251-286.

http://www.scielo.org.co/scielo.php?

script=sci arttext\&pid=S0123-59312015000200016.

Navas, S. (2015). Un mercado financiero floreciente: el del dinero virtual no regulado (Especial atención a los BITCOINS). Revista CESCO de Derecho de Consumo, 13(9), 79-115. https://revista.uclm.es/index.php/cesco/article/view/717.

Ordoñez-Iriarte, J. (2020). Salud mental y salud ambiental. Una visión prospectiva. Informe SESPAS 2020. Gaceta Sanitaria, 34(1), 68-75. https://doi.org/10.1016/j.gaceta.2020.05.007.

Painemil, M., Manquenahuel S., Biso P. \& Muñoz C. (2021). Creencias versus conocimiento en futuro profesorado. Un estudio comparado sobre neuromitos a nivel internacional. Revista Electrónica Educare, 25(1), 1-22. https://www.revistas.una.ac.cr/index.php/EDUCARE/article/vi ew/11750.

Peirano, M. (2019). El enemigo conoce el sistema. Debate.

Prieto, M., Carrillo, J., \& Lucio, L. (2015). Violencia virtual y acoso escolar entre estudiantes universitarios: el lado oscuro de las redes sociales. Innovación Educativa, 15(68), 33-47. http://www.scielo.org.mx/scielo.php? script=sci arttext\&pid=S1665-26732015000200004.

Ramírez, D. (2016). Afrontando la aplicación de tecnologías a defensa y seguridad. Paseo de la Castellana: Instituto Español de Estudios Estratégicos. http://www.iee.es/Galerias/fichero/docs analisis/2016/DIEE EA57-2016 Tecnologias Seguridad DRM.pdf.

Restrepo, J. (2020). Los límites epistemológicos de las neurociencias: La falacia de las neuro-lo que sea. Revista de Psicología Universidad de Antioquia, 11(2), 201-224. https://revistas.udea.edu.co/index.php/psicologia/article/view/ 339626.

Reyes, H., Nájera, H. \& Rojo-Dominguez, A. (2009). La nanotecnología y sus riesgos: El nacimiento de la nanotoxicología. Razón y Palabra, 68(Mayo), 1-5. https://www. redalyc.org/articulo.oa?id=199520297010.

Rodríguez-Fernández, L. (2019). Desinformación y comunicación organizacional: estudio sobre el impacto de las fake news. Revista Latina de Comunicación Social, 74(13), 1714-1728. http://www.revistalatinacs.org/074paper/1406/89es. html.

Rodríguez, O., Gallego, V., Rodríguez, M. \& Ángeles del Pozo, M. (2012). Adicción a las nuevas tecnologías. Psicología de las Adicciones, 1(1), 2-6.

Rubbini, N. (5 al 7 de diciembre de 2012). Los riesgos psicosociales en el teletrabajo [Sesión de conferencia]. VII Jornadas de Sociología de la UNLP, La Plata, Argentina. http://www.memoria.fahce.unlp.edu.ar/trab eventos/ev.2237/ ev.2237.pdf.

Sagols, L. (2010). ¿Es ética la eugenesia contemporánea? Dilemata,
https://dialnet.unirioja.es/servlet/articulo?codigo=3986280.

Sandoval, I. (2016). Enfoque de la corrupción estructural: poder, impunidad y voz ciudadana. Revista Mexicana de Sociología, 78(1), 119-152. http://www.scielo.org. mx/scielo.php? script=sci arttext\&pid=S0188-25032016000100119.

Santos M., \& Fernández-Ríos, L. (2016). El factor de impacto y el futuro de las revistas académicas. El riesgo de patologización. Innovación Educativa, 16(72), 35-52. http://www.scielo.org.mx/scielo.php? script=sci arttext\&pid=S1665-26732016000300035.

Soengas-Pérez, X. \& Assif, M. (2017). El ciberactivismo en el proceso de cambio político y social en los países árabes. 
Comunicar, 25(53),

https://www.redalyc.org/articulo.oa?id=15852792005.

Tezanos, J. (2002). Desigualdad y exclusión social en las sociedades tecnológicas. Revista del Ministerio de trabajo e Inmigración, 35(2), 35-54. https://dialnet.unirioja.es/servlet/articulo? codigo $=253629$.

Thaler R. \& Sunstein, C. (2017). Un pequeño empujón. Taurus.

Turchin, A., \& Denkenberger, D. (2018). Classifcation of global catastrophic risks connected with artifcial intelligence. Al \& Society, 35(1),

147-163.

https://link.springer.com/article/10.1007/s00146-018-0845-5.
Wexler, A. (2017). The Social Context of "Do-lt-Yourself" Brain Stimulation: Neurohackers, Biohackers, and Lifehackers. Frontiers in Human Neuroscience, 11(224), 1-6. https://www.frontiersin.org/articles/10.3389/fnhum.2017.0022 4/full

Wolinsky, H. (2016). The FBI and biohackers: an unusual relationship. EMBO reports, 17(6), 793-796. https://www.ncbi.nlm.nih.gov/pmc/articles/PMC5278613/.

Yearly, S. (1993). La autoridad social de la ciencia en la edad posmoderna. Política y Sociedad, 14(15), 59-66. https://core.ac.uk/download/pdf/38819514.pdf.

\section{Cómo citar este artículo:}

Siancas, W. (2021). Retos 4.0: un manifiesto a considerar para la 4RI. Futuro Hoy, 2(1), 16-21. https://doi.org/10.5281/zenodo.4654853

Esta obra está bajo licencia internacional

Creative Commons 4.0 Reconocimiento 4.0. 